\title{
Systems with Local and Nonlocal Diffusions, Mixed Boundary Conditions, and Reaction Terms
}

\author{
Mauricio Bogoya $\mathbb{D D}^{1}$ and Julio D. Rossi $\mathbb{D}^{2}$ \\ ${ }^{1}$ Departamento de Matemáticas, Universidad Nacional de Colombia, Bogotá, Colombia \\ ${ }^{2}$ Departamento de Matemática, FCEyN, Universidad de Buenos Aires, Ciudad Universitaria, Pabellon I (1428), \\ Buenos Aires, Argentina
}

Correspondence should be addressed to Mauricio Bogoya; mbogoyal@unal.edu.co

Received 12 July 2018; Revised 24 August 2018; Accepted 13 September 2018; Published 1 October 2018

Academic Editor: Jozef Banas

Copyright (C) 2018 Mauricio Bogoya and Julio D. Rossi. This is an open access article distributed under the Creative Commons Attribution License, which permits unrestricted use, distribution, and reproduction in any medium, provided the original work is properly cited.

We study systems with different diffusions (local and nonlocal), mixed boundary conditions, and reaction terms. We prove existence and uniqueness of the solutions and then analyze global existence vs blow up in finite time. For blowing up solutions, we find asymptotic bounds for the blow-up rate.

\section{Introduction}

1.1. Description of the Problem. Our first goal in this paper is to study the following nonlocal reaction-diffusion system with mixed boundary conditions

$$
\begin{aligned}
u_{t}(x, t)= & \int_{\Omega} J(x-y)(u(y, t)-u(x, t)) d y \\
& +v^{p}(x, t), \quad x \in \Omega, t>0, \\
v_{t}(x, t)= & \int_{\mathbb{R}^{N}} J(x-y)(v(y, t)-v(x, t)) d y \\
& +u^{q}(x, t), \quad x \in \Omega, t>0, \\
v(x, t)= & 0 \quad x \notin \Omega, t>0, \\
u(x, 0)= & u_{0}(x), \\
v(x, 0)= & v_{0}(x),
\end{aligned}
$$

$x \in \Omega$.

Here $p, q>0, u_{0}(x), v_{0}(x) \in C(\bar{\Omega})$ are nonnegative and nontrivial functions, $J: \mathbb{R}^{n} \longrightarrow \mathbb{R}$ is a nonnegative, smooth, symmetric radially and strictly decreasing kernel, with $\int_{\mathbb{R}^{n}} J(x) d x=1$ and supported in the unitary ball, and
$\Omega \subset \mathbb{R}^{N}(N \geq 1)$ is a bounded connected and smooth domain.

In the first equation, we are imposing that the diffusion takes place only in $\Omega$; no mass may enter or leave the domain. This corresponds to what is called Neumann boundary conditions in the literature; see [1]. In the second equation, we have that the diffusion takes place in the whole $\mathbb{R}^{N}$ but we impose that $u$ vanishes outside $\Omega$. This is the analogous of what is called Dirichlet boundary conditions; see [1]. Hence this system is governed by nonlocal diffusion with mixed boundary conditions.

Our second objective in this paper is the study of the same kind of system when one of the diffusions is local

$$
\begin{aligned}
u_{t}(x, t)= & \Delta u+v^{p}(x, t), \quad x \in \Omega, t>0 \\
v_{t}(x, t)= & \int_{\mathbb{R}^{N}} J(x-y)(v(y, t)-v(x, t)) d y \\
& +u^{q}(x, t), \quad x \in \Omega, t>0
\end{aligned}
$$

$\frac{\partial u}{\partial \eta}(x, t)=0$,

$$
\begin{aligned}
(x, t) & \in \partial \Omega \times(0, T) ; \\
v(x, t) & =0,
\end{aligned}
$$

$x \notin \Omega, t>0$ 


$$
\begin{aligned}
& u(x, 0)=u_{0}(x), \\
& v(x, 0)=v_{0}(x),
\end{aligned}
$$$$
x \in \Omega \text {. }
$$

Again, we assume that $p, q>0, u_{0}(x), v_{0}(x) \in C(\bar{\Omega})$ are nontrivial functions, $J$ is as before, and $\Omega \subset \mathbb{R}^{N}(N \geq 1)$ is a bounded connected and smooth domain with $\eta$ denoting its outer normal unit vector field.

Now, we face a system similar to the previous one, in which one of the two components obeys a local classical diffusion (with the usual Laplacian) while the other component has a nonlocal diffusion operator.

1.2. Main Results. For both problems (1) and (2) we analyze the existence and uniqueness of nonnegative solutions. Once we settle this issue, we look for the existence of solutions globally defined in time and solutions blowing up in finite time. Finally, when we have solutions that blow up, we analyze the blow-up rate.

We say that a solution $(u, v)$ blows up in finite time if and only if a finite time $T>0$ exists such that

$$
\lim _{t>T} \sup \left(\|u(x, t)\|_{L^{\infty}(\Omega)}+\|v(x, t)\|_{L^{\infty}(\Omega)}\right)=\infty .
$$

If solutions are well defined and finite for every $t \in(0, T)$ with $T=\infty$ the solution $(u, v)$ is global, i.e., the solution exists for all $t \geq 0$. follows.

For both problems our results can be summarized as

Theorem 1. For $\left(u_{0}, v_{0}\right) \in C(\bar{\Omega}) \times C(\bar{\Omega})$ positive real functions, there exists a unique positive solution $(u, v)$. Moreover, $(u, v)$ can be extended to a maximal interval $[0, T)$ with $T \leq \infty$. holds.

A comparison principle between super- and subsolutions

If $p q>1$, the solution $(u, v)$ blows up in finite time $T$, while for $p q \leq 1$ the solution $(u, v)$ exists globally.

For solutions that attain their maximum at $x=0$ for everyt (for example, for radially symmetric nondecreasing solutions), the blow-up rates are give by

$$
\begin{aligned}
& u(0, t) \sim(T-t)^{-(p+1) /(p q-1)} \\
& v(0, t) \sim(T-t)^{-(q+1) /(p q-1)} .
\end{aligned}
$$

1.3. Previous Results. Nonlocal diffusion equations of the form

$$
\begin{aligned}
u_{t}(x, t) & =J * u-u(x, t) \\
& =\int_{\mathbb{R}^{n}} J(x-y) u(y, t) d y-u(x, t),
\end{aligned}
$$

and variations of it, have been widely used in the last decade to model diffusion processes; see [1-7] and references therein. This equation is called nonlocal diffusion equation since the diffusion of the density $u$ at a point $x$ and time $t$ depends not only on $u(x, t)$, but also on all the values of $u$ in a neighborhood of $x$ through the convolution term $J * u$. This equation shares many properties with the classical heat equation $u_{t}=\Delta u$ such as the fact that bounded stationary solutions are constant, a maximum principle holds for both of them, and even if $J$ is compactly supported, perturbations propagate with infinite speed.

Concerning boundary conditions for nonlocal diffusion, Chasseigne et al. in [5] studied the Dirichlet boundary conditions problem

$$
\begin{aligned}
& u_{t}(x, t)=\int_{\mathbb{R}^{N}} J(x-y)(u(y, t)-u(x, t)) d y, \\
& x \in \Omega, t>0, \\
& u(x, t)=0, \quad x \notin \Omega, t>0, \\
& u(x, 0)=u_{0}(x), \quad x \in \Omega,
\end{aligned}
$$

In this model they have that diffusion takes place in the whole $\mathbb{R}^{N}$ but impose that $u$ vanishes outside $\Omega$. This is the analogous of what is called Dirichlet boundary conditions for the heat equation.

Also in [5] the Neumann boundary conditions case is studied

$$
\begin{array}{ll}
u_{t}(x, t)=\int_{\Omega} J(x-y)(u(y, t)-u(x, t)) d y, & \\
& x \in \Omega, t>0 \\
u(x, 0)=u_{0}(x), \quad x \in \Omega . &
\end{array}
$$

Since we are now integrating in $\Omega$, the diffusion takes place only in $\Omega$. The individuals may not enter nor leave $\Omega$. This is the analogous of what is called homogeneous Neumann boundary conditions in the literature.

Concerning reaction terms, Pérez-Llanos and Rossi in [8] studied the problem

$$
\begin{aligned}
u_{t}(x, t)= & \int_{\Omega} J(x-y)(u(y, t)-u(x, t)) d y \\
& +u^{p}(x, t), \quad x \in \Omega, t>0, \\
u(x, 0)= & u_{0}(x), \quad x \in \Omega .
\end{aligned}
$$

They prove that nonnegative and nontrivial solutions blow up in finite time if and only if $p>1$. Moreover, they find that the blow-up rate is the same as the one that holds for the ODE $u_{t}=u^{p}$, that is, $\lim _{t \rightarrow T^{-}}(T-t)^{1 /(p-1)}\|u(\cdot, t)\|_{\infty}=(1 /(p-$ 1) $)^{1 /(p-1)}$.

For systems, Bogoya in [9] studied the analogous system to (1) with Neumann boundary conditions.

The paper is organized as follows: In Section 2, we analyze the existence and uniqueness of solutions to (1). In Section 3, we look for global existence vs blow up of solutions to (1) and its blow-up rate when appropriate. In Section 4, we deal with the existence and uniqueness of solutions to (2). Finally, in Section 5, we analyze the global existence and blow up of solutions of (2) and the corresponding blow-up rate. 


\section{Existence and Uniqueness for (1)}

In this section, we analyze the existence and uniqueness of nonnegative solutions $(u, v)$ of $(1)$.

We will deal with this issue for a slightly more general system in which the reaction terms $u^{q}, v^{p}$ are replaced by $f(v), g(u)$, two Lipschitz nonnegative functions. Existence and uniqueness will be obtained via Banach's fixed point theorem. Let $t_{0}>0$ be fixed and consider

$$
\begin{aligned}
X_{t_{0}} & =C\left(\left[0, t_{0}\right]: C(\bar{\Omega}) \times C(\bar{\Omega})\right)=\left\{(u, v):\left[0, t_{0}\right]\right. \\
& \longrightarrow C(\bar{\Omega}) \\
& \times C(\bar{\Omega}):(u, v) \text { is continuos in space and time }\},
\end{aligned}
$$

that is, a Banach space with the norm

$$
\begin{aligned}
|\|(u, v)\||=\max _{0 \leq t \leq t_{0}}\|(u(\cdot, t), v(\cdot, t))\|_{I}, & \\
I & =L^{\infty}(\bar{\Omega}) \times L^{\infty}(\bar{\Omega})
\end{aligned}
$$

and

$$
\|(u(\cdot, t), v(\cdot, t))\|_{I}=\max _{x \in \bar{\Omega}}|u(x, t)|+\max _{x \in \bar{\Omega}}|v(x, t)| .
$$

Let

$$
P_{t_{0}}=\left\{(u, v) \in X_{t_{0}}: u \geq 0, v \geq 0\right\},
$$

that is, a closed subspace of $X_{t_{0}}$. In what follows, we will use the notation

$$
\|u\|_{\mathcal{K}}=\max _{0 \leq t \leq t_{0}}\|u(\cdot, t)\|_{L^{\infty}(\bar{\Omega})} .
$$

We define the operator

$$
\begin{aligned}
\psi_{\left(u_{0}, v_{0}\right)}: P_{t_{0}} \longrightarrow & P_{t_{0}}, \\
& \text { as } \psi_{\left(u_{0}, v_{0}\right)}(u, v)=\left(T_{u_{0}}(u), S_{v_{0}}(v)\right),
\end{aligned}
$$

where

$$
\begin{aligned}
& T_{u_{0}}(u)(x, t) \\
& =\int_{0}^{t} \int_{\Omega} J(x-y)(u(y, s)-u(x, s)) d y d s \\
& \quad+\int_{0}^{t} f(v(x, s)) d s+u_{0}(x) \\
& S_{v_{0}}(v)(x, t) \\
& = \begin{cases}\int_{0}^{t} \int_{\mathbb{R}^{N}} J(x-y)(v(y, s)-v(x, s)) d y d s \\
+\int_{0}^{t} g(u(x, s)) d s+v_{0}(x) & x \in \Omega \\
0 & x \notin \Omega .\end{cases}
\end{aligned}
$$

The following Lemma is just the first step in order to show that $\psi_{\left(u_{0}, v_{0}\right)}$ has a fixed point in $P_{t_{0}}$.
Lemma 2. Let $f$ and $g$ be Lipschitz functions with Lipschitz constants $K_{1}$ and $K_{2}$ and $\left(u_{0}, v_{0}\right)$ and $\left(w_{0}, z_{0}\right)$ two pairs of initial conditions in $C(\bar{\Omega}) \times C(\bar{\Omega})$ and $(u, v),(w, z) \in P_{t_{0}}$. Then, there exists a positive constant $C=C\left(K_{1}, K_{2}, \Omega, J\right)$ such that

$$
\begin{aligned}
& \quad\left\|\psi_{\left(u_{0}, v_{0}\right)}(u, v)-\psi_{\left(w_{0}, z_{0}\right)}(w, z)\right\| \mid \\
& \quad \leq C t_{0}|\|(u, v)-(w, z)\||+\left\|\left(u_{0}, v_{0}\right)-\left(w_{0}, z_{0}\right)\right\|_{I}
\end{aligned}
$$

Proof. For any $(x, t) \in \bar{\Omega} \times\left[0, t_{0}\right]$ we have

$$
\begin{aligned}
& \left|T_{u_{0}}(u(x, t))-T_{w_{0}}(w(x, t))\right| \\
& \leq\left|u_{0}(x)-w_{0}(x)\right| \\
& \quad+\int_{0}^{t} \int_{\Omega} J(x-y)|u(y, s)-w(y, s)| d y d s \\
& \quad+\int_{0}^{t} \int_{\Omega} J(x-y)|u(x, s)-w(x, s)| d y d s \\
& \quad+\int_{0}^{t}|f(v(x, s))-f(z(x, s))| d s \\
& \leq\left\|u_{0}-w_{0}\right\|_{L^{\infty}(\bar{\Omega})} \\
& \quad+2 \int_{0}^{t}\|u(\cdot, s)-w(\cdot, s)\|_{L^{\infty}(\bar{\Omega})} d s \int_{\Omega} J(x-y) d y \\
& \left.\quad+K_{1} \int_{0}^{t} \| v(\cdot, s)-z(\cdot, s)\right) \|_{L^{\infty}(\bar{\Omega})} d s \\
& \leq\left\|u_{0}-w_{0}\right\|_{L^{\infty}(\bar{\Omega})}+\left(2 K|\Omega|+K_{1}\right) t\|u-w\|_{\mathcal{K}},
\end{aligned}
$$

where $K=\|J\|_{\infty}$ and $K_{1}$ is the Lipschitz constant of $f$.

Analogously, taking into account the fact that $v$ is zero outside $\Omega$ and denoting by $K_{2}$ the Lipschitz constant for $g$, we have that

$$
\begin{aligned}
& \left|S_{v_{0}}(v(x, t))-S_{z_{0}}(z(x, t))\right| \\
& \quad \leq\left\|v_{0}-z_{0}\right\|_{L^{\infty}(\bar{\Omega})}+\left(2 K|\Omega|+K_{2}\right) t\|v-z\|_{\mathcal{K}} .
\end{aligned}
$$

Therefore, we have obtained

$$
\begin{aligned}
& \|\| \psi_{\left(u_{0}, v_{0}\right)}(u, v)-\psi_{\left(w_{0}, z_{0}\right)}(w, z) \| \mid \\
& \leq\left(2 K|\Omega|+K_{3}\right) t_{0}|\|(u, v)-(w, z)\|| \\
& \quad+\left\|\left(u_{0}, v_{0}\right)-\left(w_{0}, z_{0}\right)\right\|_{I},
\end{aligned}
$$

where $K_{3}=\max \left\{K_{1}, K_{2}\right\}$. Then, if $C=2 K|\Omega|+K_{3}$ we have that

$$
\begin{aligned}
& \|\| \psi_{\left(u_{0}, v_{0}\right)}(u, v)-\psi_{\left(w_{0}, z_{0}\right)}(w, z) \| \mid \\
& \quad \leq C t_{0}|\|(u, v)-(w, z)\||+\left\|\left(u_{0}, v_{0}\right)-\left(w_{0}, z_{0}\right)\right\|_{I} .
\end{aligned}
$$

Next, we show the existence and uniqueness of the solution for functions $f$ and $g$ that are locally Lipschitz. 
Theorem 3. Let $f$ and $g$ be locally Lipschitz functions and $\left(u_{0}, v_{0}\right) \in C(\bar{\Omega}) \times C(\bar{\Omega})$ be nonnegative real functions; then there exists a unique solution $(u, v)$ such that $(u, v) \in P_{t_{0}}$. Moreover, $(u, v)$ can be extended to a maximal interval $[0, T)$ with $T \leq \infty$.

Proof. We check first that $\psi_{\left(u_{0}, v_{0}\right)}: P_{t_{0}} \longrightarrow P_{t_{0}}$. Taking $(w, z)=$ $\left(w_{0}, z_{0}\right)=(0,0)$ in Lemma 2 we get that $\psi_{\left(u_{0}, v_{0}\right)}(u, v) \in P_{t_{0}}$. Choose $t_{0}$ such that $C t_{0}<1$. Now taking $\left(w_{0}, z_{0}\right)=\left(u_{0}, v_{0}\right)$ in Lemma 2 we get that $\left(u_{0}, v_{0}\right)$ is a strict contraction in $P_{t_{0}}$ and the existence and uniqueness part of the theorem follow from Banach's fixed point theorem in the interval $\left[0, t_{0}\right]$. To extend the solution to $[0, T)$ we may take as initial conditions $u\left(x, t_{0}\right), v\left(x, t_{0}\right) \in C(\Omega)$ and obtain a solution up to $\left[0,2 t_{0}\right]$. Iterating this procedure, we get a solution defined in $[0, T)$.

Now, we use the same ideas of the previous analysis to obtain the following results.

Corollary 4. The solution $(u, v)$ depends continuously on the initial data. In fact, let $f$ and $g$ be locally Lipschitz functions and if $(u, v)$ and $(w, z)$ are solutions with initial data $\left(u_{0}, v_{0}\right)$ and $\left(w_{0}, z_{0}\right)$, respectively, then there exists a constant $\widetilde{C}$ such that

$$
\|(u, v)-(w, z)\| \mid \leq \widetilde{C}\left\|\left(u_{0}, v_{0}\right)-\left(w_{0}, z_{0}\right)\right\|_{I} .
$$

Corollary 5. $(u(x, t), v(x, t)) \in P_{t_{0}}$ is a solution of (1) if and only if

$$
\begin{aligned}
& u(x, t) \\
& =\int_{0}^{t} \int_{\Omega} J(x-y)(u(y, s)-u(x, s)) d y d s \\
& \quad+\int_{0}^{t} f(v(x, s)) d s+u_{0}(x) \\
& v(x, t) \\
& = \begin{cases}\int_{0}^{t} \int_{\Omega} J(x-y)(v(y, s)-v(x, s)) d y d s & x \in \Omega \\
+\int_{0}^{t} g(u(x, s)) d s+v_{0}(x) & x \notin \Omega .\end{cases}
\end{aligned}
$$
(1).

Now, we look for existence and uniqueness of solutions to

Theorem 6. If $p, q \geq 1$ and $\left(u_{0}, v_{0}\right) \in C(\bar{\Omega}) \times C(\bar{\Omega})$ are nonnegative real functions, then there exists a unique solution $(u, v)$ of $(1)$ such that $(u, v) \in P_{t_{0}}$.

Proof. The functions $f(v)=v^{p}, g(u)=u^{q}$ are locally Lipschitz; then by Theorem 3, system (1) has a unique solution $(u, v) \in P_{t_{0}}$.

For exponents less than one, we have the following existence result.
Theorem 7. Let $p, q>0$ and $\left(u_{0}, v_{0}\right) \in C(\bar{\Omega}) \times C(\bar{\Omega})$ be nonnegative and bounded functions; then there exists a solution $(u, v) \in P_{t_{0}}$ to $(1)$.

Proof. The existence of the solution of (1) is obtained with an approximation procedure. We assume that $0<p<1 \leq q$ (the other possibilities are left to the reader). Let $\left(f_{n}\right)_{n}$ be a sequence of locally Lipschitz functions such that, for $n$ fixed $f_{n}(s)=0$, if $s \leq 0, f_{n}$ is nondecreasing and $\lim _{n \rightarrow \infty} f_{n}(s)=s^{p}$ for $s \geq 0$. Consider the system with $f_{n}(v)$ and $u^{q}$. From Lemma 2 and Theorem 3, we have that there is a unique solution $\left(u_{n}(x, t), v_{n}(x, t)\right)$. Now, $\left(u_{n}(x, t)\right)_{n}$ and $\left(v_{n}(x, t)\right)_{n}$ are nondecreasing and bounded sequences. Therefore, passing to the limit as $n \longrightarrow \infty$ using Corollary 5 , we get the existence of $(u(x, t), v(x, t))$, a solution to (1).

Remark 8. For future reference, let us analyze the solution to the ODE system

$$
\begin{aligned}
& u^{\prime}(t)=v^{p}(t), \\
& v^{\prime}(t)=u^{q}(t) \\
& \quad \text { for } t>0 \\
& u(0)=a \geq 0, \\
& v(0)=b \geq 0 .
\end{aligned}
$$

If $p q>1$, the solution to (23) is given by

$$
\begin{aligned}
u(t) & =C_{1}\left(\frac{(p q-1)^{(p+1) /(p q-1)}}{\left((p+1)(q+1)^{p}\right)^{1 /(p q-1)} a^{(p q-1) /(p+1)}}\right. \\
-t & )^{-(p+1) /(p q-1)}, \\
v(t) & =C_{2}\left(\frac{(p q-1)^{(q+1) /(p q-1)}}{\left((p+1)^{q}(q+1)\right)^{1 /(p q-1)} b^{(p q-1) /(q+1)}}\right. \\
-t & ,
\end{aligned}
$$

with

$$
\begin{aligned}
& C_{1}=\left(\frac{(p+1)(q+1)^{p}}{(p q-1)^{(p+1)}}\right)^{1 /(p q-1)} \text { and } \\
& C_{2}=\left(\frac{(p+1)^{q}(q+1)}{(p q-1)^{(q+1)}}\right)^{1 /(p q-1)} .
\end{aligned}
$$

If $p q<1$, the solution of $(23)$ is

$$
\begin{aligned}
& u(t)=\left(a^{(1-p q) /(p+1)}+C_{1} t\right)^{(p+1) /(1-p q)}, \\
& v(t)=\left(b^{(1-p q) /(q+1)}+C_{2} t\right)^{(q+1) /(1-p q)},
\end{aligned}
$$


with

$$
\begin{aligned}
& C_{1}=\left(\frac{1-p q}{p+1}\right)\left(\frac{p+1}{q+1}\right)^{p /(p+1)} \text { and } \\
& C_{2}=\left(\frac{1-p q}{q+1}\right)\left(\frac{q+1}{p+1}\right)^{q /(q+1)} .
\end{aligned}
$$

If $p q=1$, the solution of $(23)$ is

$$
\begin{aligned}
u(t) & =a e^{C_{1} t}, \\
C_{1} & =\left(\frac{p+1}{q+1}\right)^{p /(p+1)} ; \\
v(t) & =b e^{C_{2} t}, \\
C_{2} & =\left(\frac{q+1}{p+1}\right)^{q /(q+1)} .
\end{aligned}
$$

We will use the notation $(a, b) \geq(c, d)$ to indicate that $a \geq c$ and $b \geq d$.

Definition 9. Let $\bar{u}, \bar{v} \in C^{1}([0, T) ; C(\bar{\Omega})) .(\bar{u}, \bar{v})$ is called a supersolution of (1) if

$$
\begin{aligned}
\bar{u}_{t}(x, t) \geq & \int_{\Omega} J(x-y)(\bar{u}(y, t)-\bar{u}(x, t)) d y \\
& +\bar{v}^{p}(x, t), \quad x \in \Omega, t>0, \\
\bar{v}_{t}(x, t) \geq & \int_{\mathbb{R}^{N}} J(x-y)(\bar{v}(y, t)-\bar{v}(x, t)) d y \\
& +\bar{u}^{q}(x, t), \quad x \in \Omega, t>0, \\
v(x, t) \geq & 0, \quad x \notin \Omega, t>0, \\
\bar{u}(x, 0) \geq & u_{0}(x), \\
\bar{v}(x, 0) \geq & v_{0}(x),
\end{aligned}
$$

$x \in \Omega$.

Analogously $(\underline{u}, \underline{v}) \in P_{t_{0}}$ is called a subsolution of (1) if it satisfies the opposite inequalities.

Lemma 10 (comparison principle). Let $(\underline{u}, \underline{v})$ and $(\bar{u}, \bar{v})$ be a subsolution and supersolution of (1), respectively. If $\left(\underline{u}_{0}(x), \underline{v}_{0}(x)\right) \leq\left(\bar{u}_{0}(x), \bar{v}_{0}(x)\right)$ for all $x \in \Omega$, then

$$
(\underline{u}(x, t), \underline{v}(x, t)) \leq(\bar{u}(x, t), \bar{v}(x, t))
$$

for all $(x, t) \in \Omega \times(0, T)$.
Proof. Let $w(x, t)=\bar{u}(x, t)-\underline{u}(x, t), z(x, t)=\bar{v}(x, t)-\underline{v}(x, t)$. Assume first that $w(x, 0), z(x, 0)>0$ for $x \in \Omega$. We observe that $w$ and $z$ verify

$$
\begin{aligned}
w_{t}(x, t) \geq & \int_{\Omega} J(x-y)(w(y, t)-w(x, t)) d y \\
& +\left(\frac{\bar{v}^{p}(x, t)-\underline{v}^{p}(x, t)}{\bar{v}(x, t)-\underline{v}(x, t)}\right) z(x, t), \\
z_{\mathrm{t}}(x, t) \geq & \int_{\Omega} J(x-y)(z(y, t)-z(x, t)) d y \\
& +\left(\frac{\bar{u}^{q}(x, t)-\underline{u}^{q}(x, t)}{\bar{u}(x, t)-\underline{u}(x, t)}\right) w(x, t) .
\end{aligned}
$$

Now, set $0<\delta=\min \{w(x, 0), z(x, 0)\}$ and suppose that the conclusion of the Lemma does not hold. Thus, let $t_{1}$ be the first time such that

$$
\frac{\delta}{2}=\min \left\{w\left(x, t_{1}\right), z\left(x, t_{1}\right)\right\} .
$$

We can assume that $w$ attains the previous minimum. At that time, there must be a point $x_{1} \in \Omega$ such that $w\left(x_{1}, t_{1}\right)=\delta / 2$. But, on the one hand $w_{t}\left(x_{1}, t_{1}\right) \leq 0$ and, on the other hand

$$
\begin{aligned}
w_{t}\left(x_{1}, t_{1}\right) \geq & \int_{\Omega} J\left(x_{1}-y\right)\left(w(y, t)-w\left(x_{1}, t_{1}\right)\right) d y \\
& +\left(\frac{\bar{v}^{p}\left(x_{1}, t_{1}\right)-\underline{v}^{p}\left(x_{1}, t_{1}\right)}{\bar{v}\left(x_{1}, t_{1}\right)-\underline{v}\left(x_{1}, t_{1}\right)}\right) z\left(x_{1}, t_{1}\right) \\
\geq & p \eta^{p-1}\left(x_{1}, t_{1}\right) z\left(x_{1}, t_{1}\right)>0,
\end{aligned}
$$

where $\underline{v}\left(x_{1}, t_{1}\right)<\eta\left(x_{1}, t_{1}\right)<\bar{v}\left(x_{1}, t_{1}\right)$. This gives a contradiction. Using the continuity of solutions of (1) with respect to the initial condition and an approximation argument, the result follows for general initial condition.

Corollary 11. Let $(\bar{u}, \bar{v}) \in P_{t_{0}}$ be a supersolution of (1). Then, if $\left(u_{0}, v_{0}\right) \geq(0,0)$ for $x \in \Omega$ we have that $(\bar{u}(x, t), \bar{v}(x, t)) \geq$ $(0,0)$ for all $(x, t) \in \Omega \times(0, T)$ and moreover, a strict inequality holds if $\left(u_{0}, v_{0}\right)$ is positive.

The following lemma gives us the existence of a maximal solution of (1) when $p q<1$. Its proof is analogous to those given in [10]; therefore we omit the details here.

Lemma 12. Let $0<p q<1$. Then there exists $\left(u_{M}(x, t), v_{M}(x, t)\right)$, a maximal solution of $(1)$, in the sense that if $(u(x, t), v(x, t))$ is any other solution there holds

$$
\begin{aligned}
& u(x, t) \leq u_{M}(x, t), \\
& v(x, t) \leq v_{M}(x, t)
\end{aligned}
$$

in $\Omega \times(0, \infty)$.

Moreover, $u_{M}(x, t)>0, v_{M}(x, t)>0$ for all $(x, t) \in \Omega \times(0, \infty)$.

The following theorems deal with the uniqueness problem for (1) in the cases of identically null initial condition and of nontrivial initial condition, respectively. Their proofs are analogous to those given in [9]; hence we omit them. 
Theorem 13. Let $\left(u_{0}, v_{0}\right)=(0,0)$.

(1) If $p q \geq 1$, then the unique solution of $(1)$ is $(u, v)=$ $(0,0)$.

(2) If $p q<1$, then there exists exactly one solution $(u, v)$ of (1) such that $u$ and $v$ are strictly positives.

Theorem 14. If $\left(u_{0}, v_{0}\right) \neq(0,0)$, then the solution of problem (1) is unique.

\section{Global Existence vs Blow Up for Solutions to (1)}

In this section, we look for conditions that ensure that solutions of (1) blow up in finite time or are globally defined.

Theorem 15. Let $p q>1$ and $u_{0}, v_{0} \in C(\bar{\Omega})$ be nonnegative and nontrivial functions. Then, the solution $(u, v)$ of $(1)$ blows up in finite time $T$.

Proof. Let $p q>1$ and $u_{0}(x), v_{0}(x) \in C(\bar{\Omega})$ be nonnegative and nontrivial functions and $(u, v)$ solution of (1). We define the functions for $t \geq 0$

$$
\begin{aligned}
& M(t)=\int_{\Omega} u(x, t) d x, \\
& N(t)=\int_{\mathbb{R}^{N}} \phi_{1}(x) v(x, t) d x,
\end{aligned}
$$

where $C_{1} \leq \phi_{1} \leq C_{2}$ is eigenfunction of the nonlocal operator, which is positive in $\bar{\Omega}$; see [7].

As $p \geq 1$, by Holder's inequality we have that

$$
\begin{aligned}
N^{p}(t) & =\left(\int_{\mathbb{R}^{N}} \phi_{1}(x) v(x, t) d x\right)^{p} \\
& \leq C_{2}^{p}\left(\int_{\Omega} v(x, t) d x\right)^{p} \\
& \leq C_{2}^{p}|\Omega|^{p / p^{\prime}} \int_{\Omega} v^{p}(x, t) d x \\
& \leq C \int_{\Omega} v^{p}(x, t) d x
\end{aligned}
$$

with $1 / p+1 / p^{\prime}=1$. Now, differentiating $M$ with respect to $t$ and using (1) we obtain that

$$
M^{\prime}(t)=\int_{\Omega} u_{t}(x, t) d x=\int_{\Omega} v^{p}(x, t) d x \geq C N^{p}(t)
$$

As $q \geq 1$, by Holder's inequality we have that

$$
\begin{aligned}
\int_{\Omega} \phi_{1}(x) u^{q}(x, t) d x & \geq C_{1} \int_{\Omega} u^{q}(x, t) d x \\
& \geq C_{1}|\Omega|^{q / q^{\prime}}\left(\int_{\Omega} u(x, t) d x\right)^{q} \\
& =C M^{q}(t) .
\end{aligned}
$$

Now, taking the derivative of $N$ with respect to $t$ we have that

$$
\begin{aligned}
& N^{\prime}(t)=\int_{\mathbb{R}^{N}} \phi_{1}(x) v_{t}(x, t) d x=\int_{\mathbb{R}^{N}} \phi_{1}(x) \\
& \quad \cdot \int_{\mathbb{R}^{N}} J(x-y)(v(y, t)-v(x, t)) d y \\
& \quad+\int_{\mathbb{R}^{N}} \phi_{1}(x) u^{q}(x, t) d x=\int_{\mathbb{R}^{N}} v(x, t) \phi_{1}(x) \\
& \quad \cdot \int_{\mathbb{R}^{N}} J(x-y)\left(\phi_{1}(y)-\phi_{1}(x)\right) d y \\
& +\int_{\mathbb{R}^{N}} \phi_{1}(x) u^{q}(x, t) d x \\
& \quad=-\lambda_{1} \int_{\mathbb{R}^{N}} \phi_{1}(x) v(x, t) d x \\
& +\int_{\Omega} \phi_{1}(x) u^{q}(x, t) d x \geq-\lambda_{1} N(t)+C M^{q}(t) .
\end{aligned}
$$

Summarizing, we get that

$$
\begin{aligned}
& M^{\prime}(t) \geq C N^{p}(t), \\
& N^{\prime}(t) \geq-\lambda_{1} N(t)+C M^{q}(t) .
\end{aligned}
$$

If $u_{0}, v_{0}$ are large, then $M, N$ blow up in finite time; therefore $(u, v)$ blow up in finite time.

In general, $(u, v)$ blow up in finite time for any initial data $u_{0}, v_{0}$. In fact, if we consider $\underline{u}(x, t)=a(t), \underline{v}(x, t)=b(t) \phi_{1}(x)$ for $x \in \Omega, t \geq 0$, with $a(t), \overline{b(t)}$ positive functions, then

$$
\begin{aligned}
& a^{\prime}(t)=c_{1} b^{p}(t), \\
& b^{\prime}(t)=-\lambda_{1} b(t)+c_{2} a^{q}(t) .
\end{aligned}
$$

Then, it can be obtained that $(a(t), b(t))$ (and then $(u, v)$ ) blows up in finite time.

Theorem 16. Let $p q \leq 1$ and $u_{0}(x), v_{0}(x) \in C(\bar{\Omega})$ be nonnegative and nontrivial functions. Then, the solution $(u, v)$ of (1) exists globally.

Proof. Consider $a, b$ such that $\left(u_{0}, v_{0}\right) \leq(a, b)$. Since $p q \leq 1$, by Remark 8 , we have that $(\bar{u}(t), \bar{v}(t))$; the solution of $(23)$ with $a \geq u_{0}, b \geq v_{0}$ is globally defined. Notice that $(\bar{u}(t), \bar{v}(t))$ is a supersolution of (1). If $(u(x, t), v(x, t))$ is a solution of (1) with initial conditions $\left(u_{0}(x), v_{0}(x)\right)$, then, by Lemma 10 (comparison principle), we have that $u(x, t) \leq \bar{u}(t)$ and $v(x, t) \leq \bar{v}(t)$ in $\Omega \times(0, T)$. Therefore, any solution of (1) can be continued for all times in the case $p q \leq 1$.

Next, we analyze the blow-up rate of the solutions of (1). We assume that $x=0 \in \Omega$ and note that for smooth radially symmetric and nondecreasing initial conditions (that is, when $u_{0}(r), v_{0}(r)$ are $C^{1}$ such that $u_{0}^{\prime}(r) \leq 0, v_{0}^{\prime}(r) \leq$ $0)$ the solutions are also radially symmetric and radially nondecreasing (that is, it holds that $u_{r}(r, t) \leq 0$ and $v_{r}(r, t) \leq$ $0)$. Hence, for every $t \in(0, T)$, the maximum of both components is attained at $x=0$. We state this result as follows. 
Lemma 17. If $\Omega=B(0, R)$ is a ball and $\left(u_{0}, v_{0}\right)$ are smooth, radially symmetric, and nondecreasing initial conditions (i.e. $u_{0}(r), v_{0}(r)$ are $C^{1}$ such that $\left.u_{0}^{\prime}(r) \leq 0, v_{0}^{\prime}(r) \leq 0\right)$, then both components of the solution are radially symmetric and radially nondecreasing (they verify $u_{r}(r, t) \leq 0$ and $v_{r}(r, t) \leq 0$ for every $r \in[0, R)$ and every $t>0)$.

Proof. For a proof we refer to Lemma 4.1 in [8].

Theorem 18. For $p q>1$, let $(u, v)$ be a positive solution to (1) such that the maximum is attained at $x=0$ for everyt $\in(0, T)$. Then, there exist $C_{1}, C_{2}, C_{3}, C_{4}$ positive constants such that

$$
\begin{gathered}
C_{1}(T-t)^{-(p+1) /(p q-1)} \leq u(0, t) \\
\leq C_{2}(T-t)^{-(p+1) /(p q-1)} \\
C_{3}(T-t)^{-(q+1) /(p q-1)} \leq v(0, t) \\
\leq C_{4}(T-t)^{-(q+1) /(p q-1)},
\end{gathered}
$$

where T stands for the blow-up time of the solution.

Proof. As $p q>1$, we have that $(u, v)$, the solution of (1), blows up in finite time (that we called $T$ ). We assumed that

$$
\begin{aligned}
& u(0, t)=\max _{x \in \bar{\Omega}} u(x, t) \text { and } \\
& v(0, t)=\max _{x \in \bar{\Omega}} v(x, t) .
\end{aligned}
$$

By (1), we have that

$$
\begin{aligned}
u_{t}(0, t)= & \int_{\Omega} J(0-y)(u(y, t)-u(0, t)) d y \\
& +v^{p}(0, t) \leq v^{p}(0, t) \\
v_{t}(0, t)= & \int_{\mathbb{R}^{N}} J(0-y)(v(y, t)-v(0, t)) d y \\
& +u^{q}(0, t) \leq u^{q}(0, t),
\end{aligned}
$$

As

$$
1=\int_{\mathbb{R}^{N}} J(\zeta) d \zeta \geq \int_{\Omega} J(\zeta) d \zeta
$$

we have that

$$
\begin{aligned}
u_{t}(0, t)= & \int_{\Omega} J(0-y)(u(y, t)-u(0, t)) d y \\
& +v^{p}(0, t) \geq-u(0, t)+v^{p}(0, t) \\
v_{t}(0, t)= & \int_{\mathbb{R}^{N}} J(0-y)(v(y, t)-v(0, t)) d y \\
& +u^{q}(0, t) \geq-v(0, t)+u^{q}(0, t) .
\end{aligned}
$$

Therefore, we get that for all $0<t<T$

$$
-u(0, t)+v^{p}(0, t) \leq u_{t}(0, t) \leq v^{p}(0, t)
$$

and

$$
-v(0, t)+u^{q}(0, t) \leq v_{t}(0, t) \leq u^{q}(0, t) .
$$

Multiplying the second inequality of $(47)$ by $u^{q}(0, t)$ and the first inequality of $(48)$ by $v^{p}(0, t)$, we obtain

$$
u_{t}(0, t) u^{q}(0, t) \leq v_{t}(0, t) v^{p}(0, t)+v^{p+1}(0, t),
$$

which is equivalent to

$$
\left(\frac{u^{q+1}}{q+1}\right)_{t}(0, t) \leq\left(\frac{v^{p+1}}{p+1}\right)_{t}(0, t)+v^{p+1}(0, t) .
$$

Multiplying the inequality by $(p+1) e^{(p+1) t}$ and integrating in $[0, t]$ with $t<T$, we have that

$$
\begin{aligned}
u^{q}(0, t) & \leq\left((q+1) e^{(p+1) t} v^{p+1}(0, t)+C\right)^{q /(q+1)} \\
& \leq\left((q+1) e^{(p+1) T} v^{p+1}(0, t)+C\right)^{q /(q+1)} \\
& \leq C(v(0, t))^{(p+1) q /(q+1)} .
\end{aligned}
$$

Replacing the second inequality of (48) by inequality (51), we get

$$
v_{t}(0, t) \leq C(v(0, t))^{(p+1) q /(q+1)} .
$$

Integrating this inequality on $[t, T)$, we finally obtain

$$
v(0, t) \geq C_{3}(T-t)^{-\beta}, \quad \text { with } \beta=\frac{q+1}{p q-1} .
$$

In an analogous way we obtain that

$$
u(0, t) \geq C_{1}(T-t)^{-\alpha}, \quad \text { with } \alpha=\frac{p+1}{p q-1} .
$$

With an analysis similar to the one developed above, we obtain that there exists a constant $C>0$ such that for $0<$ $t<T$

$$
C(v(0, t))^{(p+1) q /(q+1)} \leq u^{q}(0, t) .
$$

Replacing the first inequality of (48) by inequality (55) and as $p q>1$ we have that $(p+1) q /(q+1)>1$ and

$$
\begin{aligned}
C(v(0, t))^{(p+1) q /(q+1)} \leq & -v(0, t) \\
& +C(v(0, t))^{(p+1) q /(q+1)} \\
\leq & v_{t}(0, t) .
\end{aligned}
$$

Integrating the inequality from above on $[t, T)$, we obtain that

$$
v(0, t) \leq C_{4}(T-t)^{-\beta} .
$$

In an analogous way we get

$$
u(0, t) \leq C_{2}(T-t)^{-\alpha} .
$$




\section{Local and Nonlocal Diffusion}

In this section, we analyze the existence and uniqueness of nonnegative solutions $(u, v)$ of $(2)$.

As in Section 2, we will initially study the system with $g(u)$ and $f(v)$ replacing $u^{q}, v^{p}$, with $f, g$ being nonnegative Lipschtiz functions. Existence and uniqueness will be again obtained via Banach's fixed point theorem. Let $t_{0}>0$ be fixed; we consider the Banach space $X_{t_{0}}$ with the norm

$$
|\|(u, v)\||=\max _{0 \leq t \leq t_{0}}\|(u(\cdot, t), v(\cdot, t))\|_{I},
$$

and $P_{t_{0}}$ a closed subspace of $X_{t_{0}}$ already considered in Section 2 .

We define the operator $\omega\left(u_{0}, v_{0}\right): P_{t_{0}} \longrightarrow P_{t_{0}}$, by

$$
\omega_{\left(u_{0}, v_{0}\right)}(u, v)=\left(\varphi_{u_{0}}(u), \phi_{v_{0}}(v)\right) \text {, }
$$

where

$$
\begin{aligned}
& \varphi_{u_{0}}(u)(x, t)=S(t) u_{0}+\int_{0}^{t} S(t-s) f(v(x, s)) d s \\
& \phi_{v_{0}}(v)(x, t) \\
& =\left\{\begin{array}{cc}
\int_{0}^{t} \int_{\mathbb{R}^{N}} J(x-y)(v(y, s)-v(x, s)) d y d s \\
+\int_{0}^{t} g(u(x, s)) d s+v_{0}(x) & x \in \Omega \\
0 & x \notin \Omega,
\end{array}\right.
\end{aligned}
$$

where for any function $h \in L^{1}(\Omega)$

$$
S(t) h=\int_{\Omega} G(x, y, t) h(y) d y
$$

where $G$ is Green's function. That is, $S(t) h$ denote the solution of (see [11])

$$
\begin{gathered}
u_{t}-\Delta u=0 \quad(x, t) \in \Omega \times(0, T) \\
\frac{\partial u}{\partial \eta}(x, t)=0 \quad(x, t) \in \partial \Omega \times(0, T) ; \\
u(x, 0)=h(x), \quad x \in \Omega,
\end{gathered}
$$

Now, we need the following lemma.

Lemma 19. Let $\left(u_{0}, v_{0}\right),\left(w_{0}, z_{0}\right) \in C(\bar{\Omega}) \times C(\bar{\Omega})$ and $(u, v),(w, z) \in P_{t_{0}}$. Then, there exists a positive constant $C=$ $C\left(K_{1}, K_{2}, \Omega, J\right)$ such that

$$
\begin{aligned}
& \left|\left\|\omega_{\left(u_{0}, v_{0}\right)}(u, v)-\omega_{\left(w_{0}, z_{0}\right)}(w, z)\right\|\right| \\
& \quad \leq C t_{0}|\|(u, v)-(w, z)\||+\left\|\left(u_{0}, v_{0}\right)-\left(w_{0}, z_{0}\right)\right\|_{I} .
\end{aligned}
$$

Proof. For any $(x, t) \in \bar{\Omega}) \times\left[0, t_{0}\right]$ we have

$$
\begin{aligned}
& \left|\varphi_{u_{0}}(u(x, t))-\varphi_{w_{0}}(w(x, t))\right| \\
& \leq\left|S(t)\left(u_{0}-w_{0}\right)\right| \\
& \quad+\int_{0}^{t} \mid S(t-s)(f(v(x, s)-f(z(x, s))) \mid d s \\
& \leq C_{1}\left\|u_{0}-w_{0}\right\|_{L^{\infty}(\bar{\Omega})}+C_{2} K_{1} t\|u-w\|_{\mathcal{K}},
\end{aligned}
$$

where $K_{1}$ is the Lipschitz constant of $f$.
Taking into account that $v$ is zero outside of $\Omega$, we have that

$$
\begin{aligned}
& \left|\phi_{v_{0}}(v(x, t))-\phi_{z_{0}}(z(x, t))\right| \\
& \quad \leq\left\|u_{0}-w_{0}\right\|_{L^{\infty}(\bar{\Omega})}+\left(2 K|\Omega|+K_{2}\right) t\|u-w\|_{\mathcal{K}},
\end{aligned}
$$

where $K=\|J\|_{\infty}$ and $K_{2}$ is the Lipschitz constant of $g$.

Therefore,

$$
\begin{aligned}
& \quad\left\|\oint_{\left(u_{0}, v_{0}\right)}(u, v)-\omega_{\left(w_{0}, z_{0}\right)}(w, z)\right\| \mid \\
& \quad \leq C t_{0}|\|(u, v)-(w, z)\||+\left\|\left(u_{0}, v_{0}\right)-\left(w_{0}, z_{0}\right)\right\|_{I} .
\end{aligned}
$$

In the following theorem, we analyze the existence and uniqueness of the solution by considering that the functions $f$ and $g$ are locally Lipschitz. The proof is analogous to the one for Theorem 3; hence we omit it.

Theorem 20. Let $f$ and $g$ be locally Lipschitz functions and $\left(u_{0}, v_{0}\right) \in C(\bar{\Omega}) \times C(\bar{\Omega})$ are nonnegative real functions; then there exists a unique solution $(u, v) \in P_{t_{0}}$. Moreover, $(u, v)$ can be extended to a maximal interval $[0, T)$ with $T \leq \infty$.

With these ingredients we can show existence and uniqueness of the solution as well as a comparison principle. The proofs are analogous to the ones that we included in Section 2; hence we omit them.

Theorem 21. Let $\left(u_{0}, v_{0}\right) \in C(\bar{\Omega}) \times C(\bar{\Omega})$ be nonnegative real functions.

(i) If $p q \geq 1$ then there exists a unique solution $(u, v) \in$ $P_{t_{0}}$.

(ii) If $p q<1$ then there exists a solution $(u, v) \in P_{t_{0}}$.

Remark 22. The stationary problem

$$
\begin{aligned}
& 0=\Delta u+v^{p}, \quad x \in \Omega, \\
& 0=\int_{\mathbb{R}^{N}} J(x-y)(v(y)-v(x)) d y+u^{q},
\end{aligned}
$$

$$
\begin{array}{rr}
\frac{\partial u}{\partial \eta}(x)=0 & x \in \Omega \\
v(x)=0 & x \notin \Omega
\end{array}
$$

has a solution $u(x)=v(x)=0, x \in \Omega$.

Definition 23. Let $\bar{u}, \bar{v} \in C^{1}([0, T) ; C(\bar{\Omega})) .(\bar{u}, \bar{v})$ is called a supersolution of (2) if

$$
\begin{aligned}
\bar{u}_{t}(x, t) \geq & \Delta \bar{u}+\bar{v}^{p}(x, t), \quad x \in \Omega, t>0 \\
\bar{v}_{t}(x, t) \geq & \int_{\mathbb{R}^{N}} J(x-y)(\bar{v}(y, t)-\bar{v}(x, t)) d y \\
& +\bar{u}^{q}(x, t), \quad x \in \Omega, t>0
\end{aligned}
$$




$$
\begin{aligned}
\frac{\partial \bar{u}}{\partial \eta}(x, t) & \geq \delta>0 \quad x \in \partial \Omega, t>0 \\
\bar{v}(x, t) & \geq 0 \quad x \notin \Omega, t>0 \\
\bar{u}(x, 0) & \geq u_{0}(x) \\
\bar{v}(x, 0) & \geq v_{0}(x)
\end{aligned}
$$

$x \in \Omega$.

Analogously $(\underline{u}, \underline{v}) \in P_{t_{0}}$ is called a subsolution of (2) if it satisfies the opposite inequalities.

Lemma 24 (comparison principle). Let $(\underline{u}, \underline{v})$ and $(\bar{u}, \bar{v})$ be a subsolution and supersolution of (2), respectively. If $\left(\underline{u}_{0}(x), \underline{v}_{0}(x)\right) \leq\left(\bar{u}_{0}(x), \bar{v}_{0}(x)\right)$ for all $x \in \Omega$, then $(\underline{u}(x, t), \underline{v}(x, t)) \leq(\bar{u}(x, t), \bar{v}(x, t))$ for all $(x, t) \in \Omega \times(0, T)$.

\section{Global Existence and Blow Up for the Local/Nonlocal Diffusion System}

In this section, we analyze under what conditions the solutions of (2) blow up in finite time or are global.

Reasoning analogously to Theorems 15 and 16, we have the following theorem on the global existence and blow up of the solutions of (2); again we omit their proofs.

Theorem 25. Let $u_{0}, v_{0} \in C(\bar{\Omega})$ be nonnegative and nontrivial functions.

(1) If $p q>1$, then the solution $(u, v)$ of (2) blows up in finite time $T$.

(2) If $p q \leq 1$, then the solution $(u, v)$ of (2) exists globally.

Next, we analyze the blow-up rate of the solutions of (2). We assume that $x=0 \in \Omega$.

Theorem 26. Let $p q>1$ and $u_{0}(x), v_{0}(x) \in C(\bar{\Omega})$ be nonnegative and nontrivial functions. Let $(u, v)$ be the solution such that the maximum is reached at $x=0$ for every $t \in(0, T)$. Then, there exist $C_{1}, C_{2}, C_{3}, C_{4}$ positive constants such that

$$
\begin{gathered}
C_{1}(T-t)^{-(p+1) /(p q-1)} \leq u(0, t) \\
\leq C_{2}(T-t)^{-(p+1) /(p q-1)} \\
C_{3}(T-t)^{-(q+1) /(p q-1)} \leq v(0, t) \\
\leq C_{4}(T-t)^{-(q+1) /(p q-1)} .
\end{gathered}
$$

Proof. As $p q>1$, we have that $(u, v)$, the solution of (1), blow up in finite time $T$. Let $u(0, t)=\max _{x \in \bar{\Omega}} u(x, t)$ and $v(0, t)=$ $\max _{x \in \bar{\Omega}} v(x, t)$. By (1), we have that

$$
\begin{aligned}
u_{t}(0, t)= & \Delta u(0, t)+v^{p}(0, t) \leq v^{p}(0, t) \\
v_{t}(0, t)= & \int_{\mathbb{R}^{N}} J(0-y)(v(y, t)-v(0, t)) d y \\
& +u^{q}(0, t) \leq u^{q}(0, t)
\end{aligned}
$$

and we have that

$$
\begin{aligned}
u_{t}(0, t) \geq & \delta v^{p}(0, t) \\
v_{t}(0, t)= & \int_{\mathbb{R}^{N}} J(0-y)(v(y, t)-v(0, t)) d y \\
& +u^{q}(0, t) \geq-v(0, t)+u^{q}(0, t) .
\end{aligned}
$$

Therefore, we have that for all $0<t<T$

$$
\delta v^{p}(0, t) \leq u_{t}(0, t) \leq v^{p}(0, t)
$$

and

$$
-v(0, t)+u^{q}(0, t) \leq v_{t}(0, t) \leq u^{q}(0, t) .
$$

Multiplying the second inequality of $(73)$ by $u^{q}(0, t)$ and the first inequality of $(74)$ by $v^{p}(0, t)$, we have that

$$
u_{t}(0, t) u^{q}(0, t) \leq v_{t}(0, t) v^{p}(0, t)+v^{p+1}(0, t),
$$

which is equivalent to

$$
\left(\frac{u^{q+1}(0, t)}{q+1}\right)_{t} \leq\left(\frac{v^{p+1}(0, t)}{p+1}\right)_{t}+v^{p+1}(0, t) .
$$

From this point the proof follows analogously to what was done in the proof of Theorem 18.

\section{Data Availability}

No data were used to support this study.

\section{Conflicts of Interest}

The authors declare that they have no conflicts of interest.

\section{References}

[1] F. Andreu-Vaillo, J. M. Mazón, J. . Rossi, and J. J. ToledoMelero, Nonlocal Diffusion Problems, vol. 165 of Mathematical Surveys and Monographs, American Mathematical Society, Real Sociedad Matematica EspaNola, Madrid, Providence, RI, USA, 2010.

[2] P. W. Bates, P. C. Fife, X. Ren, and X. Wang, "Traveling waves in a convolution model for phase transitions," Archive for Rational Mechanics and Analysis, vol. 138, no. 2, pp. 105-136, 1997.

[3] M. Bogoya, "A nonlocal nonlinear diffusion equation in higher space dimensions," Journal of Mathematical Analysis and Applications, vol. 344, no. 2, pp. 601-615, 2008.

[4] C. Cortazar, M. Elgueta, and J. D. Rossi, "A nonlocal diffusion equation whose solutions develop a free boundary," Annales Henri Poincaré. A Journal of Theoretical and Mathematical Physics, vol. 6, no. 2, pp. 269-281, 2005.

[5] E. Chasseigne, M. Chaves, and J. D. Rossi, "Asymptotic behavior for nonlocal diffusion equations," Journal de Mathématiques Pures et Appliquées, vol. 86, no. 3, pp. 271-291, 2006.

[6] P. Fife, "Some nonclassical trends in parabolic and paraboliclike evolutions," in Trends in Nonlinear Analysis, pp. 153-191, Springer, Berlin, Germany, 2003. 
[7] J. Garcia-Melian and J. D. Rossi, "On the principal eigenvalue of some nonlocal diffusion problems," Journal of Differential Equations, vol. 246, no. 1, pp. 21-38, 2009.

[8] M. Pérez-Llanos and J. D. Rossi, "Blow-up for a non-local diffusion problem with Neumann boundary conditions and a reaction term," Nonlinear Analysis. Theory, Methods \& Applications. An International Multidisciplinary Journal, vol. 70, no. 4, pp. 1629-1640, 2009.

[9] M. Bogoya, "On non-local reaction-diffusion system in a bounded domain," Boundary Value Problems, Paper No. 38, 16 pages, 2018.

[10] M. Escobedo and M. A. Herrero, "A semilinear parabolic system in a bounded domain," Annali di Matematica Pura ed Applicata. Serie Quarta, vol. 165, pp. 315-336, 1993.

[11] C. Bandle and H. Brunner, "Blow-up in diffusion equations," Journal of Computational and Applied Mathematics, vol. 97, no. 1-2, pp. 3-22, 1998. 


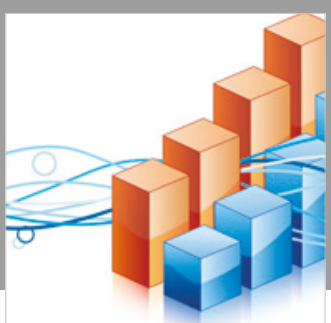

Advances in

Operations Research

\section{-n-m}
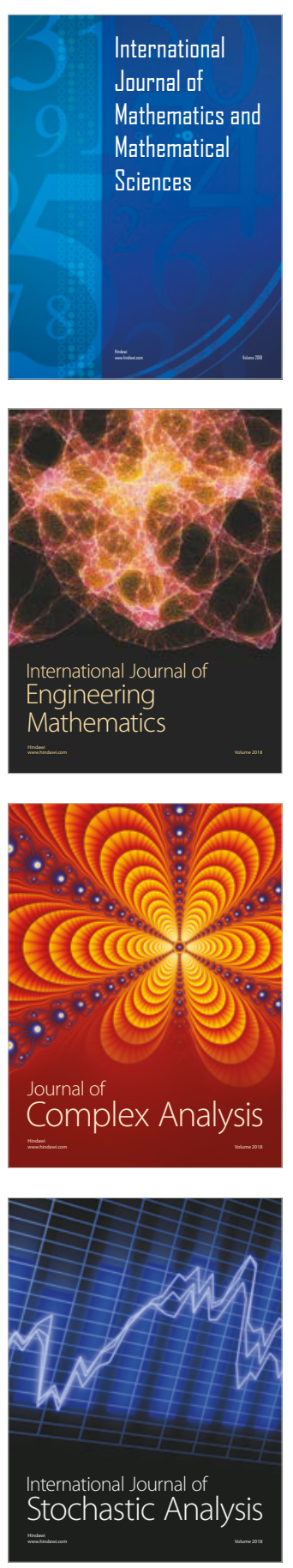
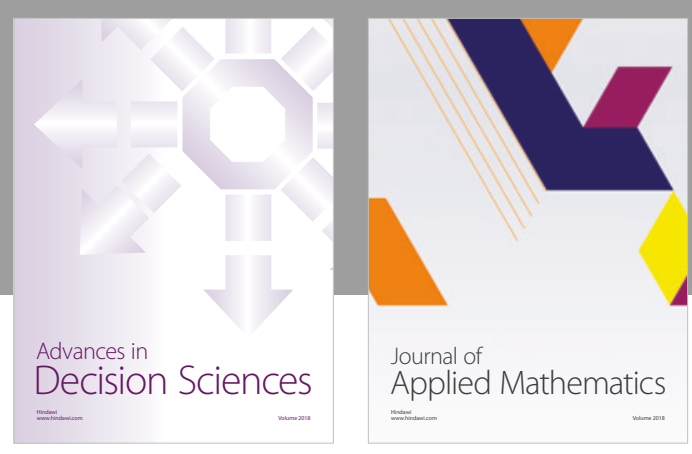

Journal of

Applied Mathematics
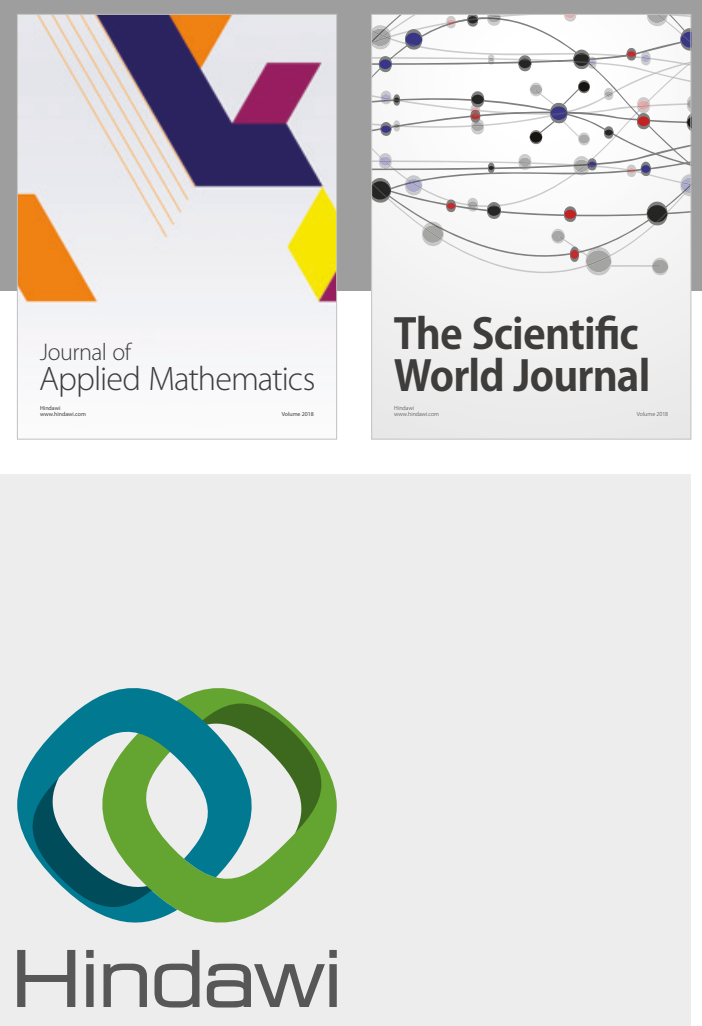

Submit your manuscripts at

www.hindawi.com

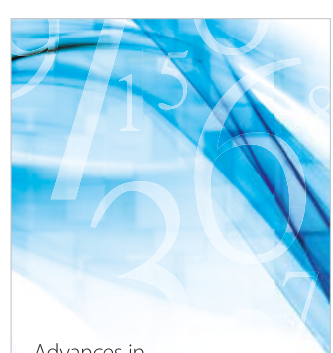

Advances in
Numerical Analysis
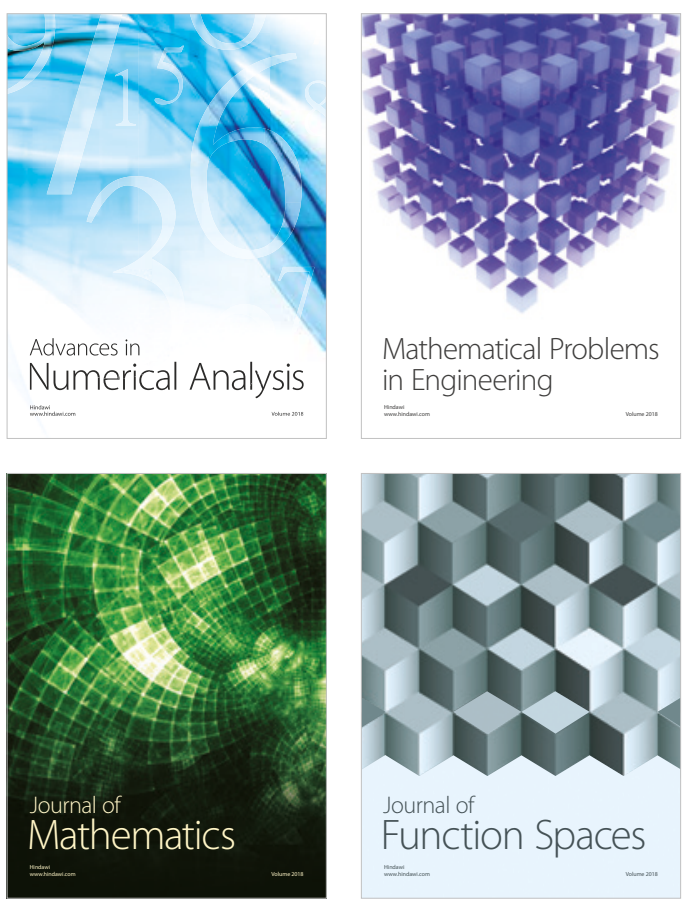

Mathematical Problems in Engineering

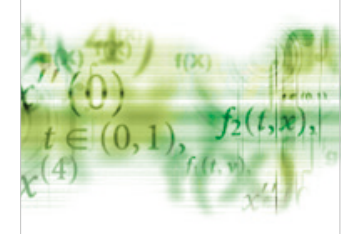

International Journal of

Differential Equations

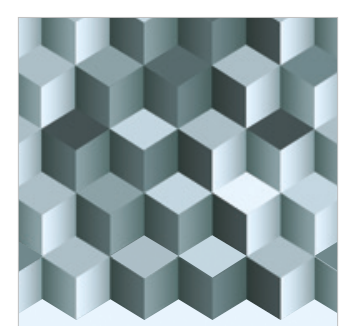

Journal of

Function Spaces

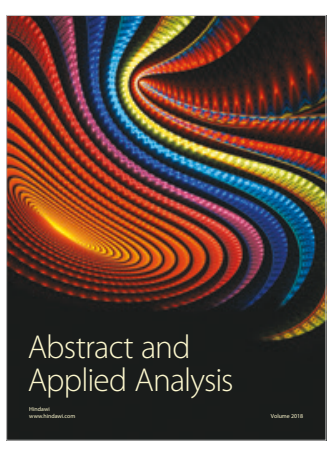

The Scientific

World Journal

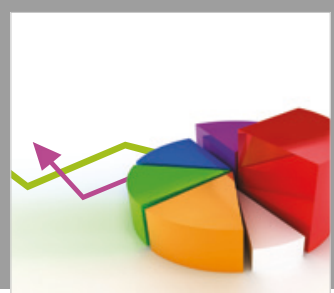

Journal of

Probability and Statistics
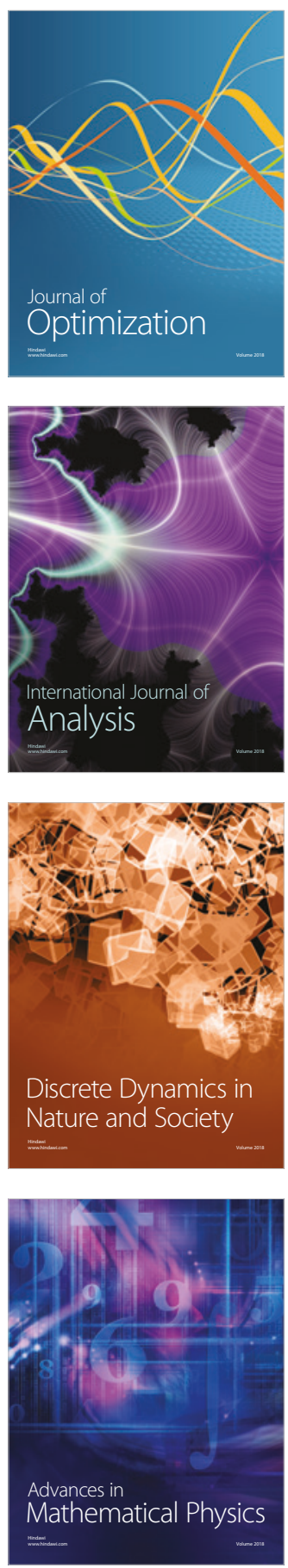